\title{
The stochastic Precise Integration method for random analysis of nonlinear systems
}

\author{
Xiaoke Gao ${ }^{1, a}$, Zhiyin Zeng ${ }^{1, b}$ Pengke Liu ${ }^{1, c}$ and Xiaojun Shao ${ }^{1, d}$ \\ ${ }^{1}$ Northwest Institute of Mechanical and Electrical Engineering, Xianyang 712099, Shaanxi, China \\ axiaokgao@163.com, 'bzy202@126.com, 'Liupengke202@126.com, 'shaoxjnwpu@163.com,
}

Keywords: stochastic process, nonlinear system, Precise Integration Method, White noise; Random excitation

Abstract. Based on deterministic Precise Integration formulation, a stochastic Precise Integration algorithm is proposed and developed in this paper for the random analysis of nonlinear systems. The recurrence relations are derived to calculate the covariance matrix response of linear and nonlinear systems subjected to stationary and non-stationary random disturbance, respectively. Numerical simulations are carried out to demonstrate the accuracy and effectiveness of the method.

\section{Introduction}

Characterization of the response of stochastic systems has long interested researchers. The dynamic response of structure is assessed by displacement, velocity, and acceleration vectors. These quantities are answers of system differential equations, formulated by dynamic equilibrium in each degree of freedom. For linear systems subjected to additive Gaussian white or linearly filtered Gaussian white noise, the response is known to be Gaussian, and it can be calculated in many ways. But for most cases, the nonlinearity of system taken into account can not be neglected. The study of the dynamic behavior of nonlinear systems subjected to stochastic excitations is of great importance in reliability analysis in engineering practice.

Recently, direct stochastic integration schemes have attracted more and more interest. H.J. Pradlwarter [1] introduced a algorithm for the computation of the covariance matrix of the stochastic response of linear and non-linear structures. A series of research work also has been done by To CWS on this filed. In his research, the stochastic central difference method [2] has been suggested, followed by the stochastic Houbolt method [3] and the stochastic Newmark algorithm [4]. Miao introduced direct integration variance prediction of random response for nonlinear systems [5]. These methods mentioned above and the work in [6] are based on second order representation of the equation of motion.

In this paper, a stochastic Precise Integration Method (SPIM) is proposed and developed for calculating the random response of nonlinear systems subjected to non-stationary random excitation. The covariance matrix in state space can be obtained directly from the recursive expression.

\section{The stochastic precise integration method for MDOF linear system}

A quit general class of linear structural mechanics problems can be cast into the following equations of motion

$$
\mathbf{M}+\mathbf{C} \ll+\mathbf{K u}=\boldsymbol{f}(t)
$$

where $\mathrm{M}, \mathrm{C}$ and $\mathrm{K}$ are mass, damping and stiffness matrices; $\boldsymbol{u}$ and $\boldsymbol{f}(t)$ are acceleration , velocity, displacement and disturbance vectors, respectively. It can be assumed that the excitation is a vector of amplitude modulated Gaussian random process with zero ensemble average, $\boldsymbol{f}(t)$ can be expressed as

$$
\boldsymbol{f}(t)=\boldsymbol{\alpha}(t) \boldsymbol{\eta}(t)
$$

in which $\boldsymbol{\alpha}(t)$ is a vector of spatial distributive deterministic envelop function and $\boldsymbol{\eta}(t)$ is a continuous random process. This assumption is appropriate for the excitations such as earthquake and sea wave etc., which have the same excitation source. 
Adopting the transformation proposed by Lin and Shen[8], let

$$
\boldsymbol{p}=\mathbf{M} \ll+\mathbf{C u} / 2 \text { or } \boldsymbol{w} \approx \mathbf{M}^{-1} \boldsymbol{p}-\mathbf{M}^{-1} \mathbf{C u} / 2
$$

Equation (1) becomes

$$
\dot{\boldsymbol{p}}=-\left(\mathbf{K}-\mathbf{C M}^{-1} \mathbf{C} / 4\right) \boldsymbol{u}-\mathbf{C} \mathbf{M}^{-1} \boldsymbol{p} / 2+\boldsymbol{f}
$$

Equations (3) and (4) can be combined to give a formulation in state space

in which

$$
\ll=\mathbf{H} v+r
$$

$$
\begin{aligned}
& \boldsymbol{v}=\left[\begin{array}{l}
\boldsymbol{q} \\
\boldsymbol{p}
\end{array}\right], \quad \boldsymbol{r}=\left[\begin{array}{l}
\boldsymbol{0} \\
\boldsymbol{f}
\end{array}\right], \quad \mathbf{H}=\left[\begin{array}{ll}
\mathbf{A} & \mathbf{D} \\
\mathbf{B} & \mathbf{G}
\end{array}\right] \\
& \mathbf{A}=-\mathbf{M}^{-1} \mathbf{C} / 2, \quad \mathbf{B}=\mathbf{C} \mathbf{M}^{-1} \mathbf{C} / 4-\mathbf{K}, \\
& \mathbf{G}=-\mathbf{C M}^{-1} / 2, \quad \mathbf{D}=\mathbf{M}^{-1}, \\
& r_{p}=f, \quad r_{q}=0, q=u,
\end{aligned}
$$

Eq.(5) is called the state equation of structural dynamics response, and the solutions can be expressed as

$$
\boldsymbol{v}(t)=\mathrm{e}^{\mathbf{H} t} \boldsymbol{v}_{\boldsymbol{0}}+\int_{0}^{t} \mathrm{e}^{\mathbf{H}(t-\tau)} \boldsymbol{r}(\tau) d \tau
$$

The duration of structural dynamic response is divided into several time intervals with time step $\Delta t$ for numerical computation. The typical discrete time $t_{n}$ for numerical computation is given by $t_{n}=n \Delta t$, $n=0,1,2, \cdots$. The relationship between $\boldsymbol{v}\left(t_{n}\right)$ and $\boldsymbol{v}\left(t_{n+1}\right)$ can be expressed as follows:

$$
\boldsymbol{v}\left(t_{n+1}\right)=e^{\mathbf{H} \Delta t} \boldsymbol{v}\left(t_{n}\right)+\int_{t_{n}}^{t_{n+1}} e^{\mathbf{H}\left(t_{n+1}-\tau\right)} \boldsymbol{r}(\tau) d \tau
$$

Lets

Eq.(8) becomes

$$
\mathbf{T}(\Delta t)=e^{\mathbf{H} \Delta t}
$$

$$
\boldsymbol{v}\left(t_{n+1}\right)=\mathbf{T}(\Delta t) \boldsymbol{v}\left(t_{n}\right)+\int_{t_{n}}^{t_{n+1}} e^{\mathbf{H}\left(t_{n+1}-\tau\right)} \boldsymbol{r}(\tau) d \tau
$$

Eq. (10) is the recursive form of the state vector $v\left(t_{n+1}\right)$. For white noise excitation, by utilizing its properties, the recursive formulation of covariance matrix can be obtained directly from Eq. (10). However, steps have to be taken to simulate the continuous stochastic process using a discrete model for nonwhite noise.

\section{White noise excitations}

Multiplying Eq.(10) and its transpose and taking the ensemble average, the recurrence formula for the covariance matrix of the system can be derived as

$$
\boldsymbol{Q}(n+1)=\mathbf{T}(\Delta t) \boldsymbol{Q}(n) \mathbf{T}^{\mathrm{T}}(\Delta t)+\mathbf{T}(\Delta t) \boldsymbol{B}(n)+\boldsymbol{B}(n)^{\mathrm{T}} \mathbf{T}^{\mathrm{T}}(\Delta t)+\boldsymbol{R}(n)
$$

in which

$$
\begin{aligned}
\boldsymbol{Q}(n) & =\left\langle\boldsymbol{v}\left(t_{n}\right) \boldsymbol{v}^{\mathrm{T}}\left(t_{n}\right)\right\rangle \\
\boldsymbol{B}(n) & =\left\langle\boldsymbol{v}\left(t_{n}\right) \int_{t_{n}}^{t_{n+1}} \boldsymbol{r}^{\mathrm{T}}(\tau) \mathbf{T}^{\mathrm{T}}\left(t_{n+1}-\tau\right) d \tau\right\rangle \\
\boldsymbol{R}(n) & =\left\langle\int_{t_{n}}^{t_{n+1}} \mathbf{T}\left(t_{n+1}-\tau\right) \boldsymbol{r}(\tau) d \tau \int_{t_{n}}^{t_{n+1}} \boldsymbol{r}^{\mathrm{T}}(\tau) \mathbf{T}^{\mathrm{T}}\left(t_{n+1}-\tau\right) d \tau\right\rangle
\end{aligned}
$$

where the superscript $\mathrm{T}$ denotes the transpose and the angular brackets $<>$ denotes the ensemble average.

For amplitude modulated white noise or Gaussian white noise, the excitation vector $f(t)$ satisfies the following: 


$$
\left\langle\boldsymbol{f}\left(t_{n}\right) \boldsymbol{f}^{\mathrm{T}}\left(t_{m}\right)\right\rangle=\left\{\begin{array}{cc}
\boldsymbol{\alpha}\left(t_{n}\right) \boldsymbol{\alpha}^{\mathrm{T}}\left(t_{n}\right)\left\langle\boldsymbol{\eta}^{2}\right\rangle & m=n \\
0 & m \neq n
\end{array}\right.
$$

Thus,

Then, $\boldsymbol{R}(n)$ can be expressed as follows:

$$
\left\langle\boldsymbol{r}\left(t_{n}\right) \boldsymbol{r}^{\mathrm{T}}\left(t_{m}\right)\right\rangle=\left\{\begin{array}{cc}
{\left[\begin{array}{cc}
0 & 0 \\
0 & \boldsymbol{\alpha}\left(t_{n}\right) \boldsymbol{\alpha}^{\mathrm{T}}\left(t_{n}\right)\left\langle\boldsymbol{\eta}^{2}\right\rangle
\end{array}\right],} & m=n \\
{\left[\begin{array}{ll}
0 & 0 \\
0 & 0
\end{array}\right] \quad,} & m \neq n
\end{array}\right.
$$

$$
\boldsymbol{R}(n)=\int_{t_{n}}^{t_{n+1}} \mathbf{T}\left(t_{n+1}-\tau\right) \boldsymbol{p}(\tau) \mathbf{T}^{\mathrm{T}}\left(t_{n+1}-\tau\right) d \tau
$$

where

$$
\boldsymbol{p}(\tau)=\left[\begin{array}{cc}
0 & 0 \\
0 & \boldsymbol{\alpha}\left(t_{n}\right) \boldsymbol{\alpha}^{\mathrm{T}}\left(t_{n}\right)\left\langle\boldsymbol{\eta}^{2}\right\rangle
\end{array}\right] .
$$

There are two expressions to calculate the auto-correlation of discrete Gaussian noise $\left\langle\boldsymbol{\eta}^{2}\right\rangle$. To [2] suggested the following expression

$$
\left\langle\boldsymbol{\eta}^{2}\right\rangle=2 \pi S_{0}
$$

While Zhang [9] pointed out that the results of the computation were seriously affected by the time step using the above formulation, and he suggested a modified expression as follows

$$
\left\langle\boldsymbol{\eta}^{2}\right\rangle=2 \pi S_{0} / \Delta t
$$

where $S_{0}$ is the spectral density of white noise. The latter expression is based on the matching of the input energy of both continuous white noise and discrete white noise. $\boldsymbol{B}(n)$ can be determined completely from (10) without any presumptions. It will be derived in the following procedure.

Multiplying Eq. (10) by $\int_{t_{n+1}}^{t_{n+2}} \boldsymbol{r}^{\mathrm{T}}(\tau) \boldsymbol{T}^{\mathrm{T}}\left(t_{n+2}-\tau\right) d \tau$ leads to

$$
\begin{aligned}
\boldsymbol{v}\left(t_{n+1}\right) \int_{t_{n+1}}^{t_{n+2}} \boldsymbol{r}^{\mathrm{T}}(\tau) \boldsymbol{T}^{\mathrm{T}}\left(t_{n+2}-\tau\right) d \tau= & \boldsymbol{T}(\Delta t) \boldsymbol{v}\left(t_{n}\right) \int_{t_{n+1}}^{t_{n+2}} \boldsymbol{r}^{\mathrm{T}}(\tau) \boldsymbol{T}^{\mathrm{T}}\left(t_{n+2}-\tau\right) d \tau+\cdots \\
& \int_{t_{n}}^{t_{n+1}} e^{\mathbf{H}\left(t_{n+1}-\tau\right)} \boldsymbol{r}(\tau) d \tau \int_{t_{n+1}}^{t_{n+2}} \boldsymbol{r}^{\mathrm{T}}(\tau) \boldsymbol{T}^{\mathrm{T}}\left(t_{n+2}-\tau\right) d \tau
\end{aligned}
$$

Expressing $\boldsymbol{v}\left(t_{n}\right)$ on the right-hand side of Eq. (21) by using Eq. (10) continuously until the state vector becomes $\boldsymbol{v}\left(t_{0}\right)$ and taking into account that $\left\langle\boldsymbol{r}\left(t_{n}\right) \boldsymbol{r}^{\mathrm{T}}\left(t_{m}\right)\right\rangle=\mathbf{0}(\mathrm{m} \neq \mathrm{n})$, Eq. (21) is changed to the following:

$$
\begin{aligned}
\boldsymbol{v}\left(t_{n+1}\right) \int_{t_{n+1}}^{t_{n+2}} \boldsymbol{r}^{\mathrm{T}}(\tau) \boldsymbol{T}^{\mathrm{T}}\left(t_{n+2}-\tau\right) d \tau \\
\quad=\boldsymbol{T}^{n+1}(\Delta t) \boldsymbol{v}\left(t_{0}\right) \int_{t_{n+1}}^{t_{n+2}} \boldsymbol{r}^{\mathrm{T}}(\tau) \boldsymbol{T}^{\mathrm{T}}\left(t_{n+2}-\tau\right) d \tau+\boldsymbol{r}\left(t_{n+1}\right) \boldsymbol{r}^{\mathrm{T}}\left(t_{n+1}\right) \boldsymbol{T}^{\mathrm{T}}(\Delta t)
\end{aligned}
$$

By applying initial conditions $\boldsymbol{v}\left(t_{0}\right)$ which is constants, Eq. (22) becomes

$$
\boldsymbol{v}\left(t_{n+1}\right) \int_{t_{n+1}}^{t_{n+2}} \boldsymbol{r}^{\mathrm{T}}(\tau) \boldsymbol{T}^{\mathrm{T}}\left(t_{n+2}-\tau\right) d \tau=\boldsymbol{r}\left(t_{n+1}\right) \boldsymbol{r}^{\mathrm{T}}\left(t_{n+1}\right) \boldsymbol{T}^{\mathrm{T}}(\Delta t)
$$

Substituting Eq. (23) at $t_{n}$ instead of $t_{n+1}$ into Eq. (13), $\boldsymbol{B}(n)$ can be derived as

$$
\boldsymbol{B}(n)=\left\langle\boldsymbol{r}\left(t_{n}\right) \boldsymbol{r}^{\mathrm{T}}\left(t_{n}\right) \boldsymbol{T}^{\mathrm{T}}(\Delta t)\right\rangle
$$

\section{Numerical examples : A Duffing oscillator}

The equation of motion for a Duffing oscillator is given as 


$$
2 \xi \alpha+\omega^{2}\left[1+\varepsilon\left(\frac{x}{\delta}\right)^{2}\right]=f(t)
$$

where

$$
\begin{aligned}
& \xi=0.1, \omega=1, \delta=52 \\
& f(t)=4\left(e^{-0.05}-e^{-0.1}\right) \eta(t) \\
& \left\langle\eta^{2}\right\rangle=2 \pi / \Delta t
\end{aligned}
$$

Fig.1 and Fig. 2 show the variance response of a Duffing oscillator using the present PIM and Monte-Carlo simulation. In Fig. $1 \varepsilon$ is chose as 0.3 and in Fig. $2 \varepsilon$ is chose as 0.5 . For such values of $\varepsilon$, the system demonstrates strong nonlinearity. It is shown that result obtained from SPIM is in good agreement with simulated data from Monte-Carlo simulation (MCS). Therefore, by employing piecewise linearization technique, the present method is accurate and efficient for systems with strong nonlinearity.
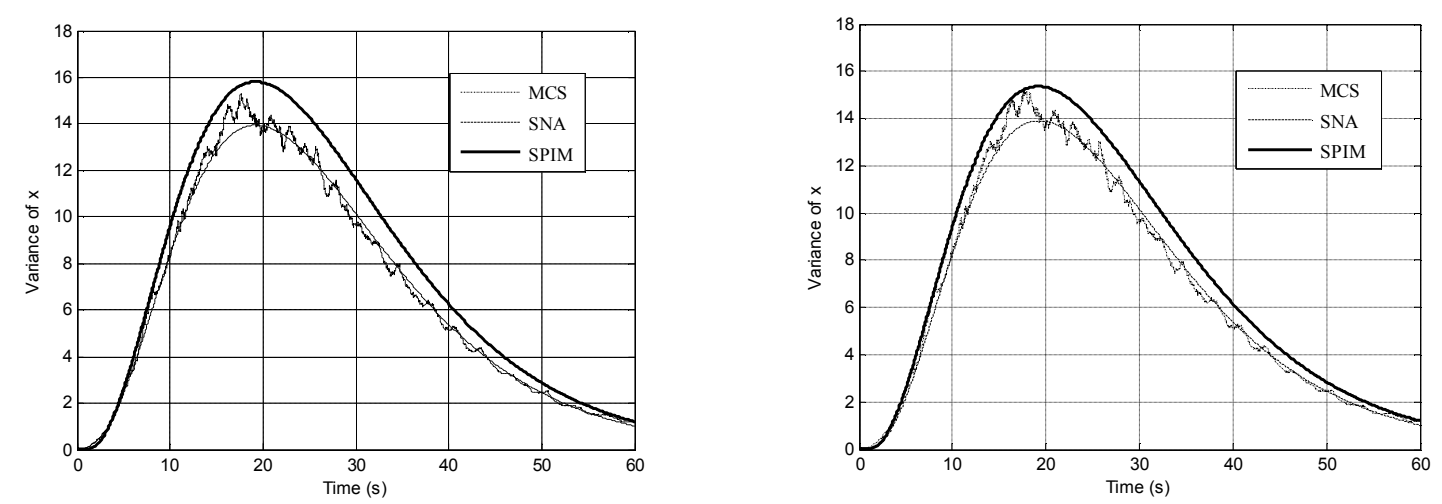

Fig. 1 Variance response of a Duffing oscillator for $\varepsilon=0.3$. Fig. 2 Variance response of a Duffing oscillator for $\varepsilon=0.5$.

\section{Summary}

In the method developed in this research, the random excitation is assumed to be the same source excitation and thus can be simplified as a single variable stochastic process. As compared to conventional methods, the proposed method is quite easy to implement. The application of the SPIM to the above examples, compared with the results obtained by stochastic Newmak algorithm (SNA) and Monte Carlo Simulation (MCS) have evidenced the good accuracy level of the method proposed in this study. In addition, the stability conditions of the developed procedure are the same as that in deterministic.

\section{References}

[1] H. J. Pradlwarter: Comput. \& Struct., Vol. 80(2002), p.1489

[2] C. W. S. To: Comput. \& Struct., Vol.23(1986), p.813

[3] C. W. S. To: Comput. \& Struct., Vol.30(1988), p.865

[4] C. W. S. To: Comput. \& Struct., Vol.44(1992), p.667

[5] Miao Blngqi: Comput. \& Struct., Vol.46(1993), p.979

[6] L. Zhang, Zu, J. W., and Zheng, Z: Comput. \& Struct., Vol.70(1999), p.557

[7] W. X. Zhong, and Williams, F. W: J. Mech. Eng. Sci. Vol.208(1994), p.427

[8] Lin Jiahao, Shen, Weiping, and Williams, F. W.: Comput. \& Struct., Vol.56(1995), p.113

[9] Zhang S. W., and Zhao, H. H.: J. Sound Vib., Vol.159(1992), p.182 